\title{
Increased Bone Density
}

National Cancer Institute

\section{Source}

National Cancer Institute. Increased Bone Density. NCI Thesaurus. Code C99124.

A finding indicating an increased density of minerals in the bones. Causes include osteopetrosis and Paget disease. 